\title{
Abstract: Augmented Reality im Operationssaal
}

\author{
Stephan Vedder \\ Universität Heidelberg, mbits imaging $\mathrm{GmbH}$ \\ vedder@mbits.info
}

Die Integration präoperativer Bilddaten in den Arbeitsablauf im Operationssaal ist eine Herausforderung, die mit immer leistungsfähigeren Bildgebungsverfahren an Dringlichkeit gewinnt. Sie steht unter der Randbedingung, Bildinformationen kontextspezifisch ohne Störung des Eingriffs bereitzustellen.

Experimentelle Augmented-Reality-Techniken, bei denen präoperative Daten auf 2D-Live-Kamerabilder gerendert werden, leiden daran, dass die Erfassung der Lage des Patienten komplexe Trackingsysteme, sowie multimodale Marker erfordert, die zwischen Bildgebung und Eingriff tage- oder wochenlang am Patienten verbleiben müssen.

Durch die Nutzung einer Tiefenkamera, ergibt sich die Möglichkeit für eine markerlose Registrierung. Anhand der gewonnenen Tiefeninformationen lässt sich eine Transformation durch Surface Matching Algorithmen bestimmen. Dazu werden die von der Tiefenkamera erzeugten Tiefendaten mit den Volumendaten einer prä- oder intraoperativen Bildgebung registriert. Anhand der berechneten Transformation ist eine passgenaue Darstellung der Bilddaten auf das reguläre Kamerabild möglich.

In dieser Arbeit wurde für die Erfassung von Farb- und Tiefenbild eine Intel Realsense Kamera verwendet. Damit das Tiefenbild für den Matching Vorgang nutzbar wird, muss daraus in mehreren Vorverarbeitungsschritten eine Punktwolke berechnet werden. Für den Matching Vorgang wurden mehrere Matching Algorithmen getestet und verglichen. Dabei konnte festgestellt werden, dass die Fast Point Feature Histogramme in Kombination mit einem RANSAC Algorithmus die besten Ergebnisse hinsichtlich Performanz und Genauigkeit liefert. Auf einem durchschnittlichen Laptop können bereits mehr als 3 Registrierungen pro Sekunde bei hoher Genauigkeit berechnet werden (weniger als $5 \mathrm{~mm}$ Translationsfehler bei einem rauschfreien Tiefenbild). Da der Matching Vorgang sehr rechenintensiv ist, kann nicht für jeden Videoframe eine Transformation berechnet werden. Um nichtsdestoweniger eine gute Darstellung zu erhalten, werden die berechneten Ergebnisse durch einen Kalman-Filter geglättet. Des Weiteren ist die Darstellung des Volumens in Echtzeit ebenfalls eine anspruchsvolle Aufgabe, weshalb dieser Vorgang optimiert wurde. Dazu wird das Volumen über Vorverarbeitung auf der GPU genau auf den benötigten Bereich beschränkt.

Es ist geplant, dass eingesetzte System im Rahmen der Eingriffsplanung in der minimal invasiven Nierentumorentfernung zu evaluieren. Ziel dieser Evaluation wird die Machbarkeit einer Zugangsplanung mit dem vorgestellten ARSystem sein. 